\title{
CLASSICAL AND QUANTUM INFORMATION PROCESSING IN ANEURAL TO NEURAL CELLULAR DECISION MAKING ON EARTH AND PERHAPS BEYOND
}

\author{
Kevin B. Clark, Ph.D. \\ (kbclarkphd@yahoo.com and kevin.clark@mpg-alumni.de)
}

Director, Felidae Conservation Fund, Mill Valley, CA 94941, USA; Co-Chairperson, Science Advisory Board, Cures Within Reach, Chicago, IL 60602, USA; Domain Champion in Biomedicine and Campus Champion, NSF Extreme Science and Engineering Discovery Environment (XSEDE), National Center for Supercomputing Applications, University of Illinois at Urbana-Champaign, Urbana, IL 61801, USA; Affiliate, NASA Ames Research Center, Mountain View, CA 94035, USA; Affiliate, NASA NfoLD, NASA Astrobiology Program, NASA Ames Research Center, Mountain View, CA 94035, USA; Advisor, Universities Space Research Association, Columbia, MD 21046, USA; Subject Matter Expert and Mentor, Penn Center for Innovation, University of Pennsylvania, Philadelphia, PA 19104, USA; Consultant and Collaborator, Research and Development Service, Veterans Affairs Greater Los Angeles Healthcare System, Los Angeles, CA 90073, USA; Main Organizer, Shared Interest Group for Natural and Artificial Intelligence (sigNAI), Max Planck Alumni Association, 14057 Berlin, Germany; Member, Nanotechnology and Biometrics Councils, Institute for Electrical and Electronics Engineers (IEEE), New York, NY 10016-5997, USA.

White Paper Running Head: ClarkKevinB4, Aneural to Neural Cellular Decision Making on Earth and Beyond 


\begin{abstract}
Response regulation to ambient and host environment conditions, known as cellular decision making, may be observed for all Earth cellular taxa, from primitive unicellular to sophisticated multicellular organisms. The origins and evolution of such capacities determine life viability and help shape the livability of worlds via biosphere interactions.
\end{abstract}

\title{
BACKGROUND
}

$\mathrm{Ca}^{2+}$-dependent cellular intelligence, decision making, or logics represent a major ubiquitous and diverse form of adaptive response regulation mediated by sets of single and/or multiple component or multi-analyte intracellular systems often conserved across and within taxonomic classifications of life on Earth and possibly elsewhere in the universe (1-16). Such systems contribute to expression of a wide variety of important life-sustaining phenomena at the cellular level, including, but not limited to, bioenergetics, posttranslational protein and lipid modification and transport, homeostasis, motility and fate, proliferation, extreme environment adaptation and transformation, aneural and neural plasticity, and complex social-like behaviors. Given its pivotal role in the origin and evolution of extant viruses/phage, Archaea, bacteria, fungi and molds, protozoa, and metazoa, $\mathrm{Ca}^{2+}$-dependent cellular logics systems and their constituent macromolecules, biochemical pathways, and organelles offer unmatched opportunities for better understanding fundamental aspects of fitness and selection on Earth and on comparable past, present, and future life-supporting astronomical bodies $(14,15)$. Increased study of $\mathrm{Ca}^{2+}$ dependent systems may give insights into other forms of cellular response regulation mediated by known and unknown mechanisms not reliant on $\mathrm{Ca}^{2+}$, such as additional divalent cations, and into macromolecular synthesis and function in the origins of life, into early cellular life and increasing complexity to multicellularity, and into co-evolution of life and eco-evolutionary transitions (14). However, despite these promising distinct advantages for space sciences and discovery, the importance of $\mathrm{Ca}^{2+}$-mediated cellular decision making is oftentimes overlooked in astrobiological research, particularly characterization of expressed fundamental classical and quantum information processing in aneual (e.g., microbes) to neural systems. Although such systems may not be found beyond Earth, they do provide models or a basis for extracellular life detection and should be considered in terms of significant astrobiological potential for shaping other possible extinct and extant livable worlds.

\section{CELLULAR DECISION MAKING AND MULTICOMPONENT LOGICS SYSTEMS}

All microbes, from viruses to protozoa, make decisions throughout their lifespan. They sense, interpret, and even manipulate changing internal homeostatic states and/or local ambient and host environments, often staying with the same strategy or switching between alternative strategies of differential fitness to determine, for instance, vegetative and reproductive cycles, phenotype, motility, stress resistance, stages of infection, and social cell-cell interactions. Successful strategies can increase a microbe's viability and/or fecundity and may vary with inherited life-history traits, random or directed mutations and epigenetic modifications, and traditional forms of dual-process nonassociative and associative learning and memory (1-16). Strategy acquisition, storage, modification, selection, and execution by microbes frequently require the coordination of $\mathrm{Ca}^{2+}$-dependent sensory transduction pathways, gene regulatory 
networks, membrane and intracellular transport systems, metabolism, and motility and adhesion apparati, making microbes highly complex computational agents crucial to understanding the origins and evolution of life on Earth and throughout the universe. Recognizing the dynamic goal-directed computational nature of microbe behavior and physiology, modern virologists, bacteriologists, phycologists, and protistologists now revisit the idea, first more-or-less anecdotally reported by early 20th-century scientists, that both solitary and colonial microbes exhibit degrees of cellular intelligence, decision making, or logistics. The kinds of nonsocial and social decision making evolved in microbes might not attain that observed for phylogenetically more recent eukaryotic organisms. Nevertheless, the controversial idea of self-deterministic microbial behavior has profound scientific implications. The ability of microbes to solve ecological dilemmas within a single generation or over many generations has been found to play significant roles in many contexts that affect the life and evolutionary trajectories of individual microbes, their communities, and environments, such as host-parasite and parasite-parasite interactions, mate selection; foraging, hunting, and farming; collective defenses against predators and stressors, kin recognition, quorum sensing, and social altruism and cheating (1-16).

$\mathrm{Ca}^{2+}$-mediated macromolecular logics systems underlying many of the above noted cellular response regulation phenomena arise from modular or superfamily protein structural domains that serve as evolutionary units whose members share a common evolutionary ancestor and render, via genetic duplication and recombination, functionally diverse protein repertoires encoded in genomes (14). Superfamilies show some nonuniform or heterogeneous distribution across simple prokaryote to complex eukaryote species, with greater abundance and diversity usually positively correlated with genome size, recombination, and evolution. Compared to other taxa, $\mathrm{Ca}^{2+}$-signaling toolkits of eukaryotes have experienced much more expansion and diversification, coinciding with organismal complexity and cell-type differentiation. But, the toolkit is evolving toward increased abundance and diversity of integrated, single-purpose proteins, rather than multipurpose $\mathrm{Ca}^{2+}$-binding macromolecules shared with ancestors - a trait that arguably improves the decision-tree and affector-effector capabilities of cellular logics systems in more complex organisms. Thus, $\mathrm{Ca}^{2+}$-signaling toolkits and the genes that encode and regulate them provide unique dissociating biosignatures to molecular fingerprint life origins, evolution, and complexities associated with large highly diverse phylogenetic trees containing aneural to neural taxa. Furthermore, coevolution has enabled viruses and microbes to coopt the more sophisticated eukaryotic, especially animal, host intracellular $\mathrm{Ca}^{2+}$-signaling pathways to optimize timing and effectiveness of infection stages against barriers to invasion, pathogenesis, replication, and release $(7.9,14,16)$. Such host-symbiont interactions provide a model for better understanding the signaling nature of intracellular response regulation pathways, including methods or processes used to speed-up and secure information processing from corruption and interception.

\section{EXAMPLES OF Ca ${ }^{2+}$-MEDIATED CELLULAR DECISION MAKING IN HOSTS AND PRIMITIVE INFECTIVE AGENTS}

Depending on the life-defining systematics one chooses, viruses offer a useful model for cellular life origins and evolution throughout the universe (e.g., 17) and, therefore, offer potential insights into the origins and evolution of cellular decision making and its physiologic mechanisms and adaptations. Virus-induced changes in free cytosolic $\mathrm{Ca}^{2+}$ levels facilitate virus 
adsorption, uncoating, catalysis, toxin production, structural assembly and stabilization, trafficking, and fusion and budding. $\mathrm{Ca}^{2+}$ associated alterations in virus status also selectively precipitate host cytopathologies through, among other events, retardation or induction of apoptosis, elevation of metabolic stress and reactive oxygen species production, and promotion of proinflammatory cytokine and chemokine synthesis and release $(7.9,14,16)$. Viral particles and proteins tune spatiotemporal dynamics of host free cytosolic $\mathrm{Ca}^{2+}$ concentrations by modulating $\mathrm{Ca}^{2+}$ entry from the extracellular environment, upstream first or second messengers, ion- and ATP-dependent $\mathrm{Ca}^{2+}$ pumps that sequester or extrude free cytosolic $\mathrm{Ca}^{2+}$, store-operated $\mathrm{Ca}^{2+}$ mobilization and leakage, and viral capsid/envelope and downstream host $\mathrm{Ca}^{2+}$ binding proteins and sensors. Exploitation of host $\mathrm{Ca}^{2+}$-signaling systems is a mechanism of survival and reproduction also co-evolved by prokaryotic and eukaryotic pathogens $(7.9,14,16)$. Normally bacteria evade host defenses by, for instance, usurping membrane repair systems, downregulating redox immunological responses, and mimicking proinflammatory chemokine and cytokine mobilization of hosts. Such pathogens facilitate influx of $\mathrm{Ca}^{2+}$ into macrophages during the invasion phase of infection, thereby increasing the activity of reactive nitrogen and oxygen response pathways as well as diminishing the apoptotic effects of elevated intracellular $\mathrm{Ca}^{2+}$ concentrations for the host cell. Protozoan pathogens and parasites similarly prime the host environment through toxin-activated $\mathrm{Ca}^{2+}$ influx into host cells and additional mechanisms.

\section{SOME CLASSICAL AND QUANTUM COMPUTATIONAL ASPECTS OF CELLULAR DECISION MAKING}

Importantly, mounting evidence from decades of analytical and experimental research continues to oppose the conventional tenet that quantum mechanical phenomena exert, at best, trivial influences over bioprocesses (1-4,6,8,10-13). Criticisms still tend to concentrate on the capacity of biological systems to settle or cohere into a quantum regime long enough to accomplish quantum computation. However, issues regarding quantum decoherence, the collapse of the Schrödinger wave function into a single classical or macroscopic state due to thermodynamic processes involving a system and its environment, are less problematic for cellular enzymatic processes reliant on small, thermally-shielded protein reaction sites and/or on local temperature gradients which can force cellular substrate from decoherent to coherent activity. Considering these factors, a number of substrate essential for microbial and neuronal computations are already associated with quantum performance characteristics, such as cytoskeletal lattices, the citric acid cycle and metabolism, molecular ratchets, molecule folding, synaptic boutons and vesicles, odorant receptors, and autocatalytic second-messenger cascades. Quantum effects at both informational and physical degrees of freedom thus seem to appear in every major aspect of cell structure and function, from sensory transduction to gene expression to cellular metabolism to cell motility. However, scaling of quantum effects between microscopic and large macroscopic physical states, such as that associated with entire brains and corresponding probabilistic cognitive events, remains a troublesome technical issue for even many of the above findings. One perhaps noteworthy exception may be found for single eukaryotic cells, such as protozoa, differentiated neurons, and perhaps other cellular forms, that perform natural quantum efficient quadratic speed-up in the search for appropriate store-operated $\mathrm{Ca}^{2+}$ response regulation of, among other processes, protein and lipid biosynthesis, cell energetics, stress responses, cell fate and death, synaptic plasticity, and immunoprotection (1-4,6,8,10-13). 
This sort of speed-up in cellular decision making results from spatiotemporal dynamics of inositol 1,4,5-trisphosphate receptor (IP3R)-dependent intracellular $\mathrm{Ca}^{2+}$-induced $\mathrm{Ca}^{2+}$ release and the search (or signaling) velocity of $\mathrm{Ca}^{2+}$ wave propagation (1-4,6,8,11-13). As chemical processes, such as the duration of $\mathrm{Ca}^{2+}$ mobilization, become rate-limiting over interstore distances, $\mathrm{Ca}^{2+}$ waves quadratically decrease interstore-travel time from slow saltatory to fast continuous gradients proportional to the square-root of the classical $\mathrm{Ca}^{2+}$ diffusion coefficient, matching the computing efficiency of Grover's quantum algorithm (see Figure 1). Moreover, the IP3R-scaled neuronal Grover's quantum algorithm, and perhaps the same and other forms found in microbes and plants, can contribute to surprising classical information processing over much longer intracellular distances and times common to global, multicompartmental $\mathrm{Ca}^{2+}$ signaling. This expectation, unaccounted for by standard (stochastic or deterministic) autocatalytic $\mathrm{Ca}^{2+}$ response regulation models, agrees with expression of three-agent quantum teleportation over communication channels transmitting classical bits via the information content of unperturbed entangled biomolecular states, a circumstance that expresses superdense coding and quantum molecular memory for microbe and neuronal messenger systems when respectively confronting environmental challenges and or mediating healthy and/or diseased mind-brain states. The microbial and neuronal versions of Grover's quantum algorithm thus maintains a capacity to benefit signal coincidence detection and integration, bidirectional plasticity, and other vital cell functions by rapidly selecting, ordering, and/or counting optional response regulation choices (1$4,6,8,11-13)$. Although not experimentally confirmed, similar phenomena may occur in plants, bacteria, and additional cellular life forms.

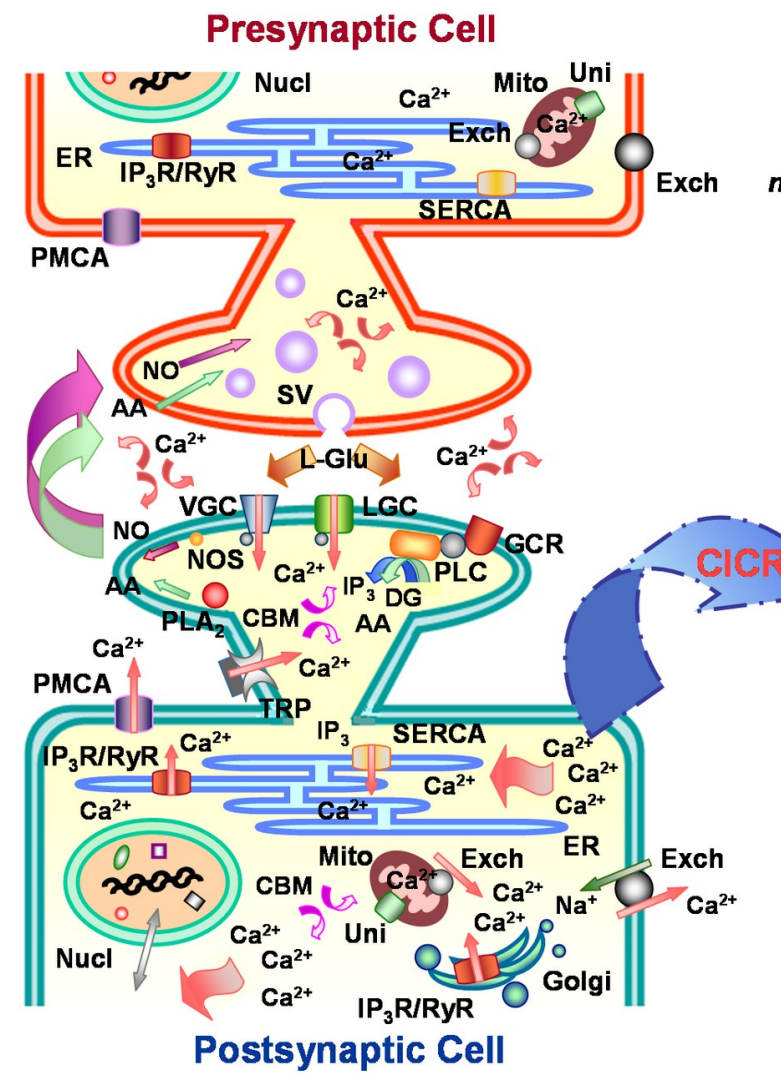

\section{Grover's Quantum Algorithm}
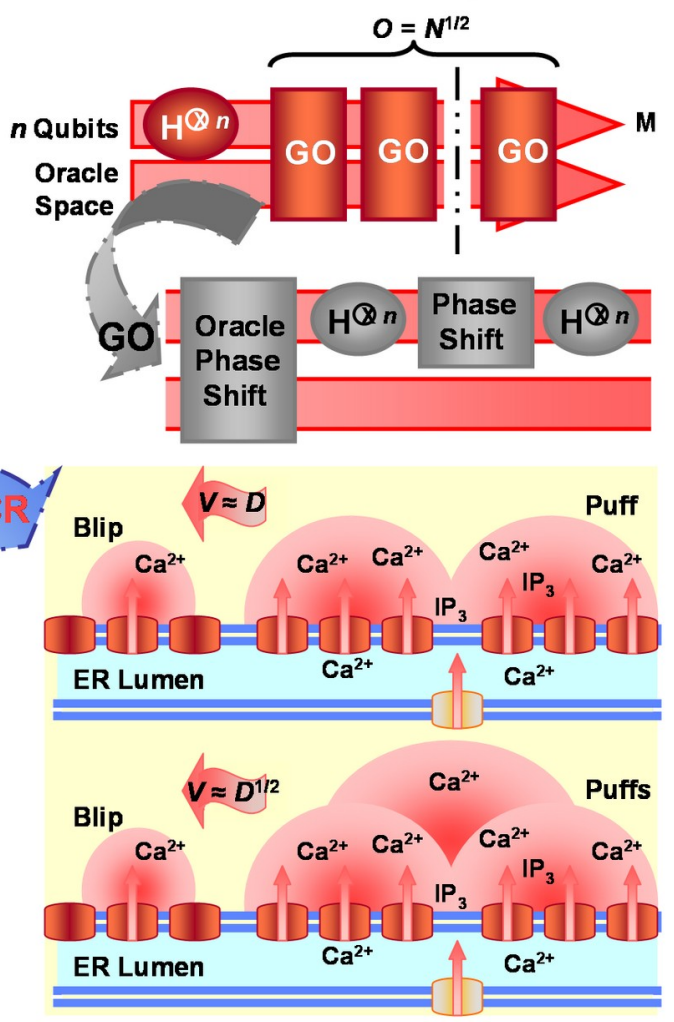
Figure 1. Calcium-induced calcium reactions (CICRs) emulate Grover's quantum algorithm in neuronal information processing - an example of eukaryotic cellular decision making. Left panel portrays major characteristic substrate (e.g., receptors, organelles, etc.) involved in $\mathrm{Ca}^{2+}$-mediated response regulation of arbitrary glutaminergic neurons, including, but not limited to, substrate critical for synaptic plasticity, cellular energetics, immunoprotection, homeostasis, gene expression, biosynthesis, molecular trafficking, cytoskeletal organization, and cell fate. Similar mechanisms affect both pre- and postsynaptic neurons, but, for descriptive purposes, postsynaptic cell activity is emphasized. $\mathrm{Ca}^{2+}$ entry into the postsynaptic neuron through voltage-gated receptor (VGC), ligand-gated receptor (LGC), and transient potential receptor (TRP) channels and stimulated inositol 1,4,5-trisphosphate $\left(\mathrm{IP}_{3}\right)$ production by activated G-protein coupled receptors (GCR) help initiate cytosolic CICRs from integral $\mathrm{IP}_{3}$ receptors $\left(\mathrm{IP}_{3} \mathrm{R}\right)$ located along the endoplasmic reticulum (ER) membrane. CICRs may cause traveling waves of varying velocities and patterns which emulate search routines capable of eliciting/suppressing appropriate response regulation from different cellular compartments. Lower right panel illustrates CICR saltatory and continuous waves. Saltatory $\mathrm{Ca}^{2+}$ waves and the information they carry conduct at velocities $(V)$ proportional to the classical $\mathrm{Ca}^{2+}$ diffusion coefficient $(D)$. Whereas, faster continuous $\mathrm{Ca}^{2+}$ waves and the information they transmit move at velocities proportional to the square-root of the classical $\mathrm{Ca}^{2+}$ diffusion coefficient. Coefficient $D$ of continuous waves for either intercluster or intracluster diffusion is assumed to be up to orders of magnitude greater than that for saltatory waves. The quadratic disparity in the velocities of saltatory and continuous waves corresponds to the root-rate increase of information processing by Grover's quantum algorithm over classical algorithms. Upper right panel shows schematic of Grover's quantum algorithm. The algorithm takes as input $n$ qubits, upon which it performs Hadamard transformations $\left(\mathrm{H}^{\otimes n}\right)$ and Grover's operation (GO) to find a target $m$ of $M$ solutions stored in database $N$. Regardless of whether one or more consultations of the Oracle are needed, Grover's quantum algorithm finds the target solution within $O=N^{1 / 2}$ algorithmic steps or operations $O$. Additional abbreviations: arachidonic acid (AA), $\mathrm{Ca}^{2+}$ binding molecule (CBM), $\mathrm{Ca}^{2+}$ uniporter (Uni), diacylgycerol (DG), Golgi apparatus (Golgi), L-glutamate (L-Glu), nucleus (Nucl), mitochondria (Mito), nitric oxide (NO), nitric oxide synthase (NOS), phospholipase $\mathrm{A}_{2}$ (PLA ${ }_{2}$ ), phospholipase $\mathrm{C}$ (PLC), plasma-membrane $\mathrm{Ca}^{2+}$ ATPase (PMCA), ryanodine receptor (RyR), sarcoplasmic-endoplasmic-reticulum $\mathrm{Ca}^{2+}$ ATPase (SERCA), $\mathrm{Na}^{+} / \mathrm{Ca}^{2+}$ exchanger (Exch), synaptic vesicle (SV). Figure 1 reproduced from (11) with permission.

\section{CONCLUSIONS}

The IP3R-based Grover's quantum algorithm of CICR behavior and $\mathrm{Ca}^{2+}$-mediated cellular response regulation imparts greater comprehensiveness than possible with traditional single receptor, intracluster, and intercluster models (11). For instance, above quantum depictions of the IP3R system uniquely permits study of computationally efficient subcellular superdense coding, quantum learning and memory, quantum error diagnosis and correction, and quantum encryption. The model nevertheless yields only a computational first-approximation of Grover's quantum algorithm and needs refinement by applying sophisticated: (1) relativistic quantum physicochemistry theory to aptly match IP3R protein structure and function with the workspaces and operators of Grover's quantum algorithm, and (2) fire-diffuse-fire or lattice-percolation mathematical treatments of intracluster IP3R activity and CICR dynamics to fully address aspects of reaction-diffusion stochasticity and cytosolic $\mathrm{Ca}^{2+}$ buffering. With respect to the latter 
topic, the model becomes particularly relevant during actuation of intracellular compartmental $\mathrm{Ca}^{2+}$ loading from ambient and host environmental cation sources. Even as a preliminary construct, the model implies contexts coincident with moderate to massive fluxes of $\mathrm{Ca}^{2+}$ through cation-permeable integral cell membrane pores and gated channels, such as during synaptic plasticity, microbial pathogen attack, and pathological cellular oxidative stress, will assist in driving cells to accelerate response regulation to quantum-level efficiency through induction of stable local and possibly subsequent global continuous $\mathrm{Ca}^{2+}$ waves.

Expression of an IP3R-based Grover's quantum algorithm, which may serve as a quantum amplifier and router for classical information, supports an estimated forty-fold boost in classical information processing by networked $\mathrm{Ca}^{2+}$ release sites through buffer-dependent superadditive $\mathrm{Ca}^{2+}$ wave densities and velocities (11). Such effects presumably enhance operational traits of, for example, signal coincidence detection and integration, bidirectional plasticity, gene expression, immunodefenses, growth and tropisms, protein modification and transport, cytoskeletal polymerization, endosome formation and other cell functions by rapidly selecting, ordering, and/or counting optional local response regulation choices. These sorts of attributes give cells powerful means to adapt to and control their environments. They also illustrate how other cellular decision-making mechanisms, perhaps mediated by the same or different metal species, might emerge and evolve under the eco-evolutionary pressures of extraterrestrial worlds, as well as exert their own eco-evolutionary pressures on the biospheres of those worlds. When considering the origins and evolution of early cellular life on Earth and perhaps elsewhere in the universe, astrobiologists should better endeavor to incorporate into their hypotheses the impact of cellular decision making.

\section{REFERENCES}

1) Clark, K.B. (2010). Origins of learned reciprocity in solitary ciliates searching grouped 'courting' assurances at quantum efficiencies. BioSystems, 99(1), 27-41.

2) Clark, K.B. (2010). Bose-Einstein condensates form in heuristics learned by ciliates deciding to signal 'social' commitments. BioSystems, 99(3), 167-178.

3) Clark, K.B. (2010). On classical and quantum error-correction in ciliate mate selection. Communicative \& Integrative Biology, 3(4), 374-378.

4) Clark, K.B. (2010). Arrhenius-kinetics evidence for quantum tunneling in microbial "social" decision rates. Communicative \& Integrative Biology, 3(6), 540-544.

5) Clark, K.B. (2012). Social biases determine spatiotemporal sparseness of ciliate mating heuristics. Communicative \& Integrative Biology, 5(1), 3-11.

6) Clark, K.B. (2013). Ciliates learn to diagnose and correct classical error syndromes in mating strategies. Frontiers in Microbiology, 4, 229.

7) Clark, K.B. (2013). Biotic activity of $\mathrm{Ca}^{2+}$-modulating nontraditional antimicrobial and -viral agents. Frontiers in Microbiology, 4, 381.

8) Clark, K.B. (2013). The mating judgments of microbes. In K.B. Clark (Ed.), Social learning theory: Phylogenetic considerations across animal, plant, and microbial taxa, pp. 173-200. Hauppauge: Nova Science Publishers, Inc. ISBN 978-1-62618-268-4.

9) Clark, K.B. and Eisenstein, E.M. (2013). Targeting host store-operated $\mathrm{Ca}^{2+}$ release to attenuate viral infections. Current Topics in Medicinal Chemistry, 13(16), 1916-1932.

10) Clark, K.B. (2014). Evolution of affective and linguistic disambiguation under social 
eavesdropping pressures. Behavioral and Brain Sciences, 37(6), 551-552.

11) Clark, K.B. (2014). Basis for a neuronal version of Grover's quantum algorithm. Frontiers in Molecular Neuroscience, 7, 29.

12) Clark, K.B. (2015). Insight and analysis problem solving in microbes to machines. Progress in Biophysics and Molecular Biology, 119, 183-193.

13) Clark, K.B. (2017). Cognitive completeness of quantum teleportation and superdense coding in neuronal response regulation and plasticity. Proceedings of the Royal Society B: Biological Sciences. eLetter. https://royalsocietypublishing.org/doi/thEsuppl/10.1098/rspb.2013.3056

14) Clark, K.B. (2018). Searching for (Proto)Cellular Logics Proteins in Earth-like Environments Hospitable and Inhospitable to Life. White paper submitted to the Committee on an Astrobiology Science Strategy for the Search for Life in the Universe, National Research Council, Washington, D.C.

15) Clark, K.B. (2019). Unpredictable homeodynamic and ambient constraints on irrational decision making of aneural and neural foragers. Behavioral and Brain Sciences, 42, e40.

16) Clark, K.B. (2019). Neurotropic enteroviruses coopt "fair-weather-friend" commensal gut microbiota to drive host infection and CNS disturbances. Behavioral and Brain Sciences, 42, e68.

17) Durzyńska, J., Goździcka-Józefiak, A. (2015). Viruses and cells intertwined since the dawn of evolution. Virology Journal, 12, 169. 